Utah State University

DigitalCommons@USU

$1-1-2010$

\title{
The Wide-Field Infrared Survey Explorer (WISE) Beamsplitter Assembly
}

\author{
Roy W. Esplin \\ Utah State University \\ D. O. Miles \\ Utah State University \\ D. K. McLain \\ Utah State University \\ H. M. Latvakoski \\ Utah State University \\ M. F. Larsen \\ Utah State University \\ M. Kahan \\ Utah State University
}

Follow this and additional works at: https://digitalcommons.usu.edu/sdl_pubs See next page for additional authors

\section{Recommended Citation}

Esplin, Roy W.; Miles, D. O.; McLain, D. K.; Latvakoski, H. M.; Larsen, M. F.; Kahan, M.; and Elwell, J. D., "The Wide-Field Infrared Survey Explorer (WISE) Beamsplitter Assembly" (2010). Space Dynamics Lab

Publications. Paper 41.

https://digitalcommons.usu.edu/sdl_pubs/41

This Article is brought to you for free and open access by the Space Dynamics Lab at DigitalCommons@USU. It has been accepted for inclusion in Space Dynamics Lab Publications by an authorized administrator of DigitalCommons@USU. For more information, please contact digitalcommons@usu.edu.

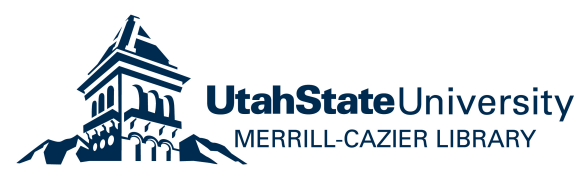




\section{Authors}

Roy W. Esplin, D. O. Miles, D. K. McLain, H. M. Latvakoski, M. F. Larsen, M. Kahan, and J. D. Elwell 


\title{
The Wide-field Infrared Survey Explorer (WISE) Beamsplitter Assembly
}

\author{
R. W. Esplin ${ }^{1}$, D. O. Miles ${ }^{1}$, D. K. McLain ${ }^{1}$, H. M. Latvakoski ${ }^{1}$, M. F. Larsen ${ }^{1}$, M. Kahan ${ }^{2}$, \\ and J. D. Elwell ${ }^{1}$ \\ ${ }^{1}$ Space Dynamics Laboratory, Utah State University, Logan, UT; \\ ${ }^{2}$ Optical Research Associates, Westborough, MA
}

\begin{abstract}
The design, fabrication and testing of the BeamSplitter Assembly (BSA) of the Wide-field Infrared Survey Explorer (WISE) instrument are discussed in the paper. The BSA splits the WISE telescope optical output beam into 4 spectral wavelength bands: 2.8-3.8, 4.1-5.2, 7.5-16.5, and 20-26 $\mu \mathrm{m}$. The BSA also provides focus adjustments to focus the WISE instrument prior to launch. The methods used to focus WISE are also discussed in this paper. Funding for and management of the WISE program were provided by the NASA Jet Propulsion Laboratory.
\end{abstract}

\section{INTRODUCTION}

The Wide Field Infrared Survey Explorer (WISE) mission ${ }^{1,2}$ and the WISE payload ${ }^{3}$ has been described in recent papers. This paper describes the WISE Beamsplitter Assembly (BSA) that splits optical output from the WISE telescope into 4 spectral bands and contains the 4 WISE focal plane assemblies (FPA). The 4 WISE spectral bands are 2.8 to $3.8 \mu \mathrm{m}, 4.1$ to $5.2 \mu \mathrm{m}, 7.5$ to $16.5 \mu \mathrm{m}$, and 20.0 to $26.0 \mu \mathrm{m}$. These bands are identified, respectively, as Bands $1,2,3$ and 4 . The WISE BSA is shown mounted on the WISE telescope in Figure 1. The Band 1,2 and 3 FPA's are identified in Figure 1;

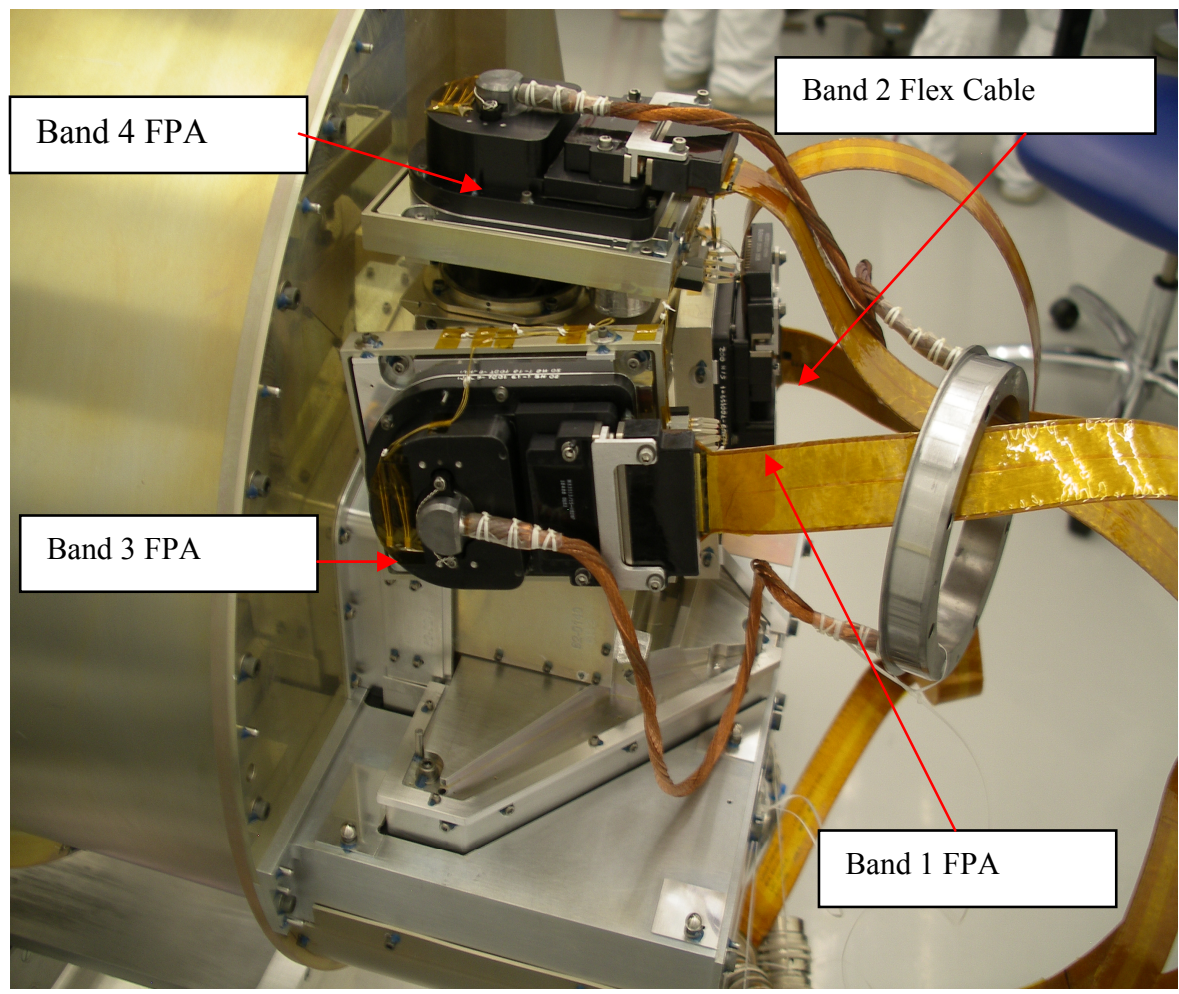
the Band 2 FPA cannot be seen in Figure 1, but the electronic flex cable going to the Band 2 FPA is identified. Figure 2 shows the BSA before installation on the telescope and before the focal plane assemblies (FPA's) were installed in the BSA. The receptacles for all 4 FPA's are clearly visible in Figure 2. The focal plane assemblies each contain a bandpass filter and a $1024 \times 1024$ detector array. The Band 1 and Band 2 detectors are $\mathrm{HgCdTe}$ operating at a temperature of $32 \mathrm{~K}$, and the Band 3 and Band 4 detector arrays are Arsenic-doped Silicon operating at a temperature of $7.3 \mathrm{~K}$. The Band 2 FPA is shown in Figure 3.

Figure 1. WISE BSA mounted on telescope. 


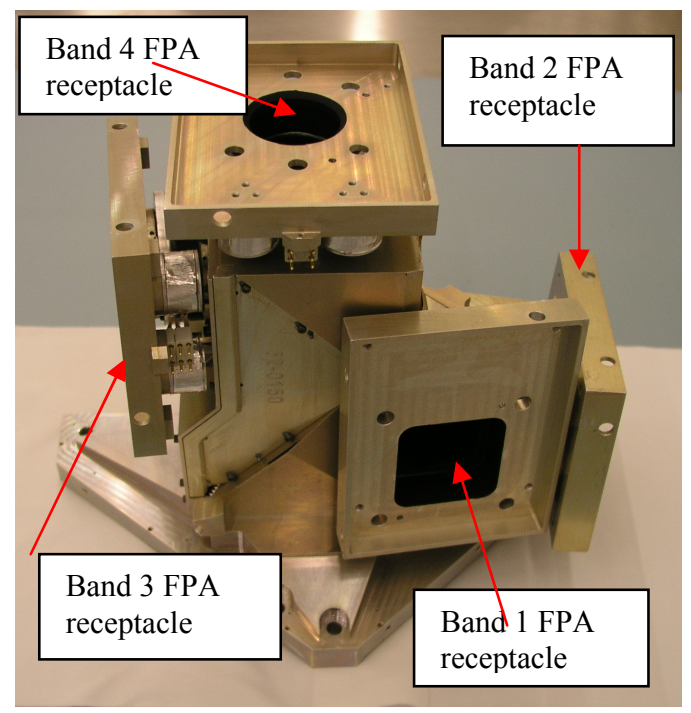

Figure 2. WISE BSA before mounting on telescope and before installation of focal plane assemblies

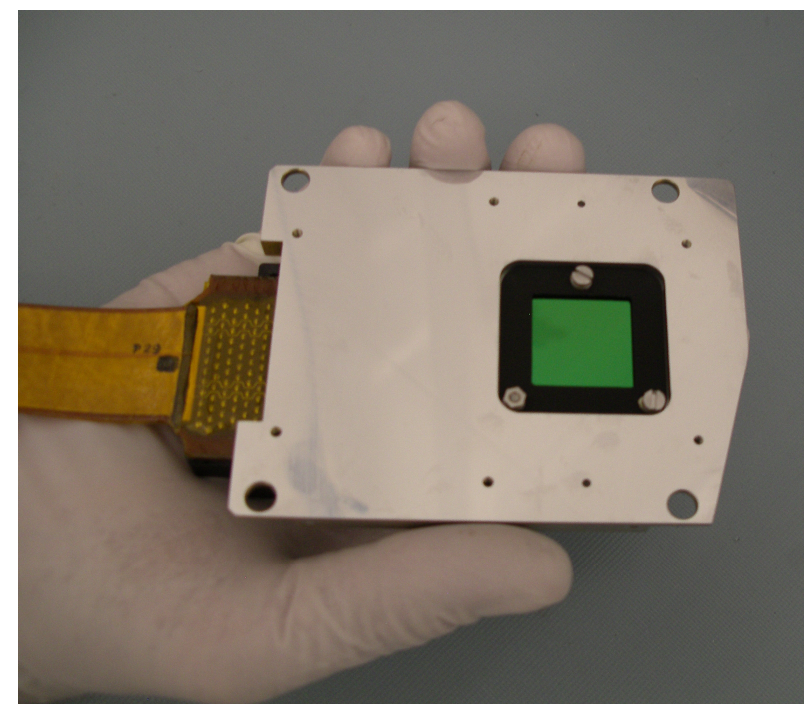

Figure 3. WISE Band 2 focal plane assembly

\subsection{WISE BSA optical-mechanical layout}

\section{WISE BSA OPTICS}

The BSA optical-mechanical layout is shown in Figure 4. Part (a) of Figure 4 shows an optical ray trace of the BSA optical components while part (b) of this figure shows a solid model of the BSA. In Figure 4 both the ray trace and the solid model are spatially oriented approximately the same. The dichroic beamsplitters and filters were made by JDSU.

\subsection{WISE BSA dichroic beamsplitters}

Since the WISE BSA operates at cryogenic temperatures, the difference between the values of the thermal coefficient of expansion for the dichroics and their mounts must be accommodated. In the WISE BSA this is done by using springloaded mounts. The WISE dichroic beamsplitters were challenging to mount because of their rectangular shape. BS1 was especially challenging because of its large size and mass. A photograph of the BS1 dichroic beamsplitter is shown in Figure 5. The dichroic mounting systems provide both axial and transverse preloading by means of springs. The cryogenic-temperature performance of the BS1 mount was verified by measuring the BS1 surface flatness error near operation temperatures in two consecutive cool downs with the spring loading set at flight preload levels. The BS1 surface error measured in two consecutive cool downs is in Figure 6. 


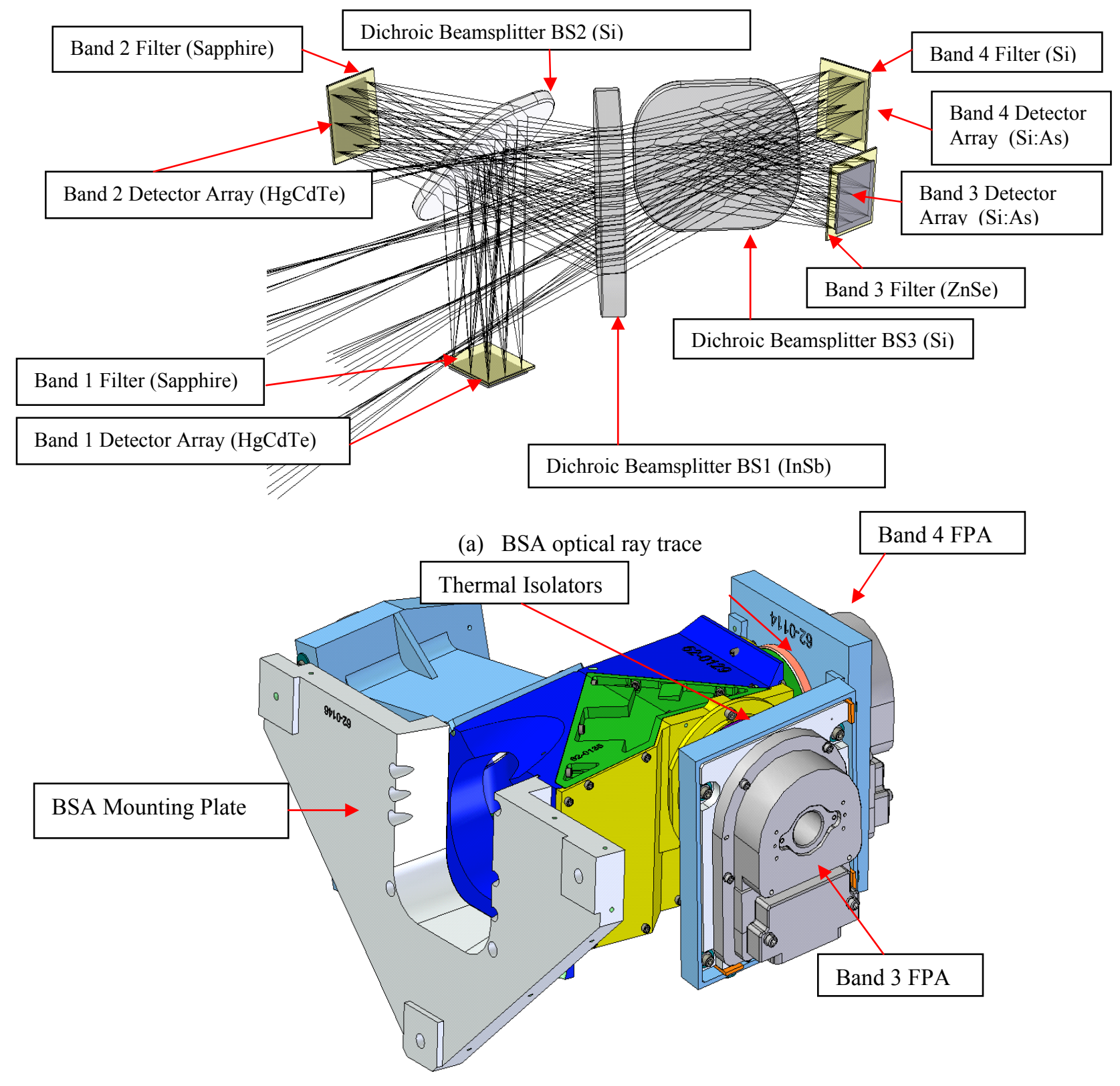

(b) BSA solid model

Figure 4. BSA optical ray trace and BSA solid model in the same spatial orientation. Substrate materials in part (a) are indicated in parentheses. 


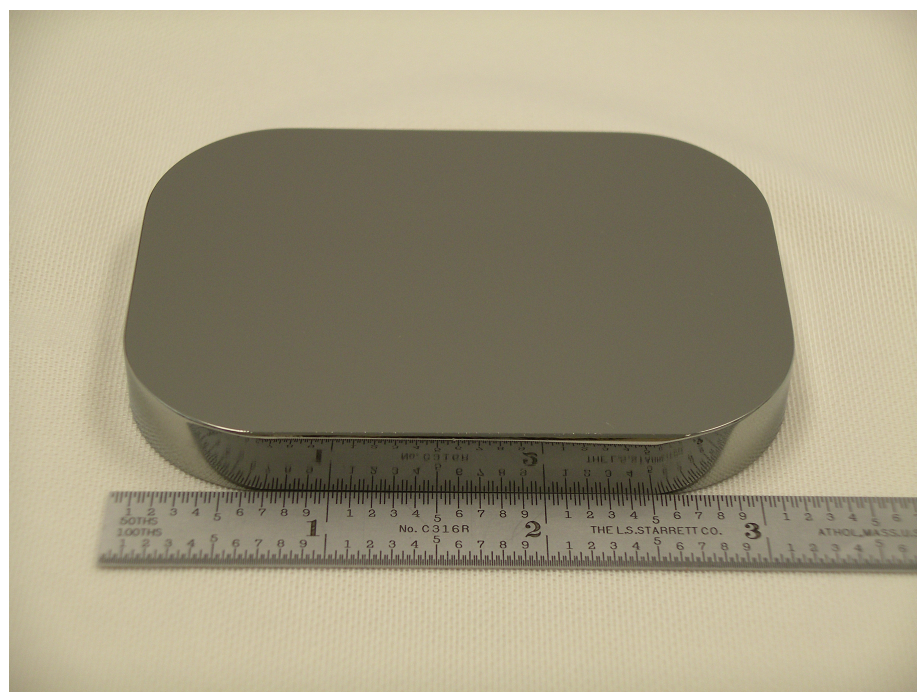

Figure 5. Photograph of InSb BS1 dichroic beamsplitter

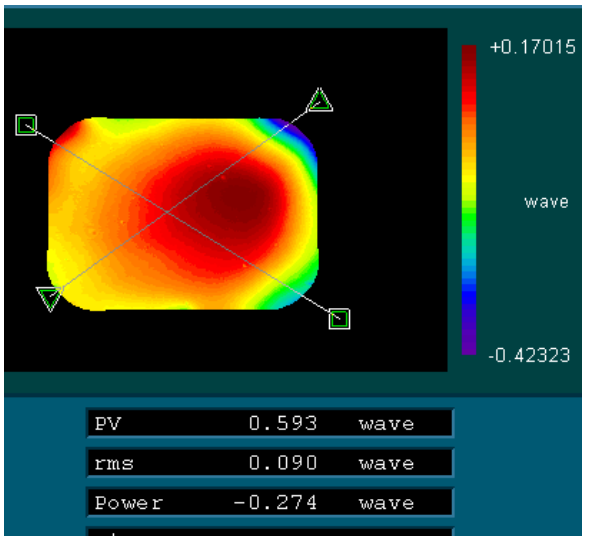

(a) First cool down (Temperature $=83 \mathrm{~K})$

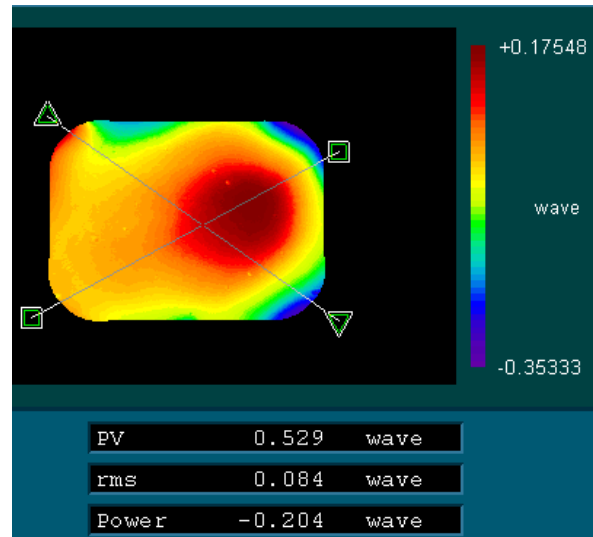

(b) Second cool down (Temperature $=82 \mathrm{~K})$

Figure 6. BS1 surface flatness errors measured in 2 successive cool downs with flight preloading at cryogenic temperatures

\subsection{Focus shims}

\section{WISE FOCUSING}

WISE was focused before flight using compound-angle wedged shims between each FPA assembly and its receptacle in the BSA. The solid model of the shim for Band 4 is shown in Figure 8. The shims were designed from measurements of the focus at the 4 corners and center of each detector array. The focus measurements used to design the focus shims were done with WISE optics inside a dewar at operation temperatures and a test collimator at ambient temperature outside the dewar. This required testing through test windows. The focus of all 4 WISE focal planes relative to each other was established first using a $\mathrm{KBr}$ test window and the all-reflective imager rear-end portion of the WISE telescope. After the afocal front-end portion of the telescope was integrated to the imager rear-end portion of the WISE telescope, the absolute focus of Band 1 was measured using a fused silica test window. The final focus shims where designed using the relative focus information from the imager measurements and the full telescope measurements of Band 1. Finally, the focus of all 4 spectral bands was verified without a test window using the Space Dynamics Laboratory (SDL) Multifunction Infrared Calibrator version 2 (MIC2). MIC2 shared a common vacuum space with the WISE instrument. 


\subsection{Mapping the FOV onto FPAs}

FOV angles were mapped to physical locations on the FPA by importing chief rays from CODE V optical program into the WISE opto-mechanical solid model. Two views of the optical-mechanical model that tie the FOV in object space to the Band 1 FPA are shown in Figure 8. The designations F1, F4, F6, F7 and F9 are the field numbers of the WISE CODE V optical model for the center, and 4 corners of the FOV. Views similar to those shown in Figure 8 were used to map the FOV to the Band 2, Band 3 and Band 4 FPAs.

\subsection{Design and fabrication of focus shims}

The first set of focus shims were made according to the nominal optical-mechanical design. Then subsequent sets of focus shims were made using defocus measurements, CMM measurements, solid modeling and computer controlled machining until the required focus was achieved.

\subsection{Focus measurement}

A conceptual ray trace of the three focus measurement setups used is shown in Figure 9. Point $C$ is the focus of the collimator so the rays from point $\mathrm{C}$ are parallel when they leave the collimator. The image of point $C$ formed by the collimator-window-WISE focus measurement setup is at point $C^{\prime}$. Similarly point $A^{\prime}$ is at the focus of the WISE optics so the rays that focus at point $A^{\prime}$ are parallel before entering the WISE instrument. The length of the line segment $\overline{A^{\prime} C^{\prime}}$ is the longitudinal defocus caused by the window. For the SDL MIC2 collimator test there was no test window so for this test $A^{\prime}$ and $C^{\prime}$ were the same point. The focus shift caused by the $\mathrm{KBr}$ test window was insignificant in the focus measurements made using only the imager portion of the WISE telescope. However, the focus shift caused by the fused silica test window that was used in the Band 1 complete telescope measurements was 0.0035 inches, which was significant. Temperature diodes on the fused silica test window and infrared images of this window indicated that for there was a thermal gradient in the fused silica test window that was approximately parabolic with the coldest point at the center of the window. This parabolic temperature gradient made the fused silica test window act as a weak divergent lens.

If the detector is at the point $B^{\prime}$, the collimator point source must be moved to the conjugate position $\mathrm{B}$ in order to focus the point image on the detector. The longitudinal distance $\overline{B C}$ was measured and multiplied by the square of the ratio of the WISE focal length to the collimator focal length in order to find the distance $\overline{B^{\prime} C^{\prime}}$. Then the WISE defocus $\overline{B^{\prime} A^{\prime}}$ was computed by subtracting the window defocus $\overline{A^{\prime} C^{\prime}}$ from $\overline{B^{\prime} C^{\prime}}$.

\section{FOCUS AND BSA VERIFICATION}

The WISE focus was verified prelaunch for all 4 bands without a test window using MIC2.

The WISE instrument met its image quality requirements on orbit in all 4 bands indicating that WISE is well focused and that the BSA assembly functions as required. The WISE on-orbit image shown in Figure 10 also indicates that the WISE instrument is well focused and that the BSA imager is doing its job well. This pseudo-color image of Andromeda is a combination of images from all 4 WISE bands. 


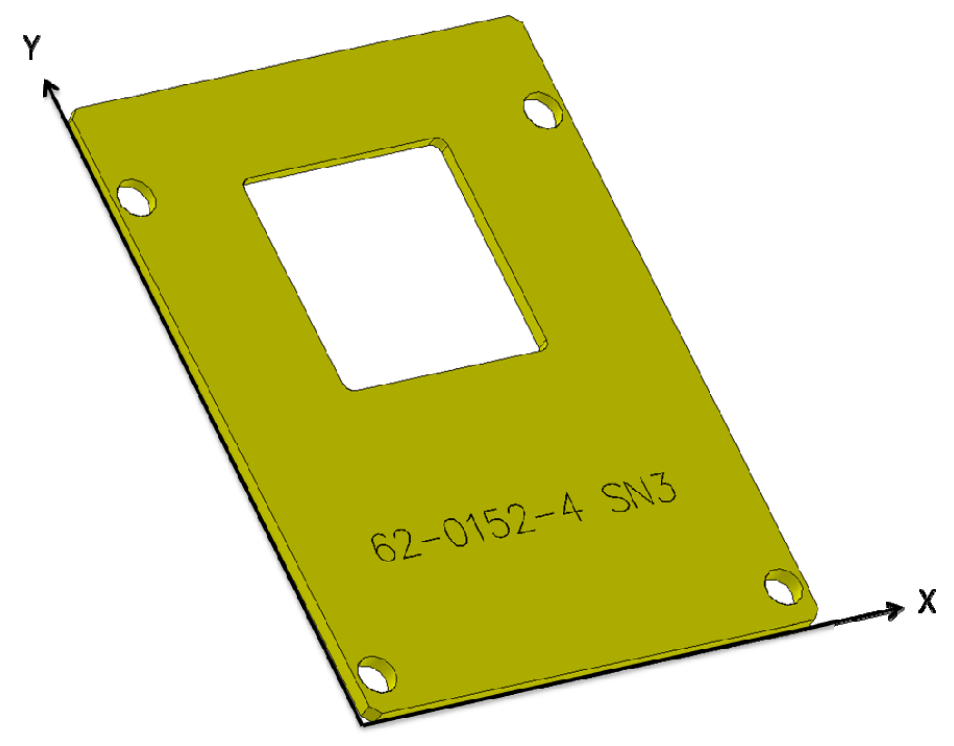

Figure 7. Solid model of Band 4 shim.

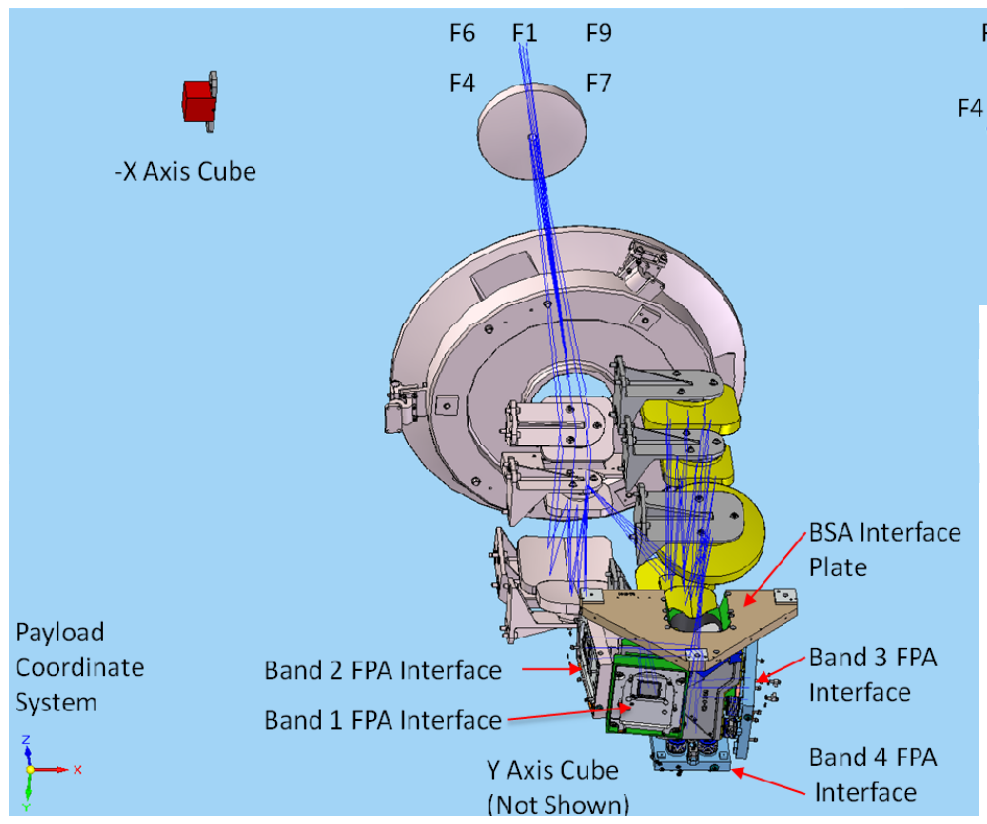

(a) Paths of chief rays through WISE optical-mechanical model

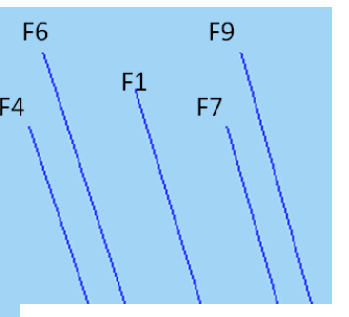

Magnified $V$ ew of Chief Rays in Object Space for Fleld Angles at Center and Corners of F'eld of View

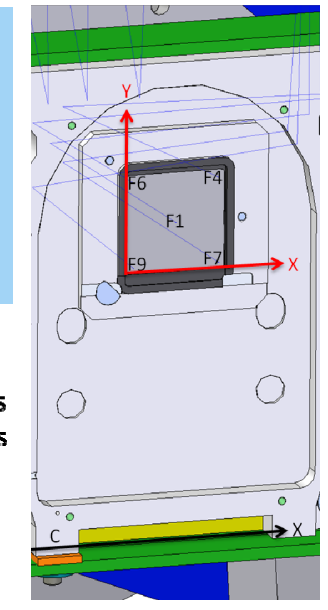

(b) Physical locations of chief rays at Band 1 FPA

Figure 8. Views of optical-mechanical model used to tie FOV to physical locations on Band 1 FPA 


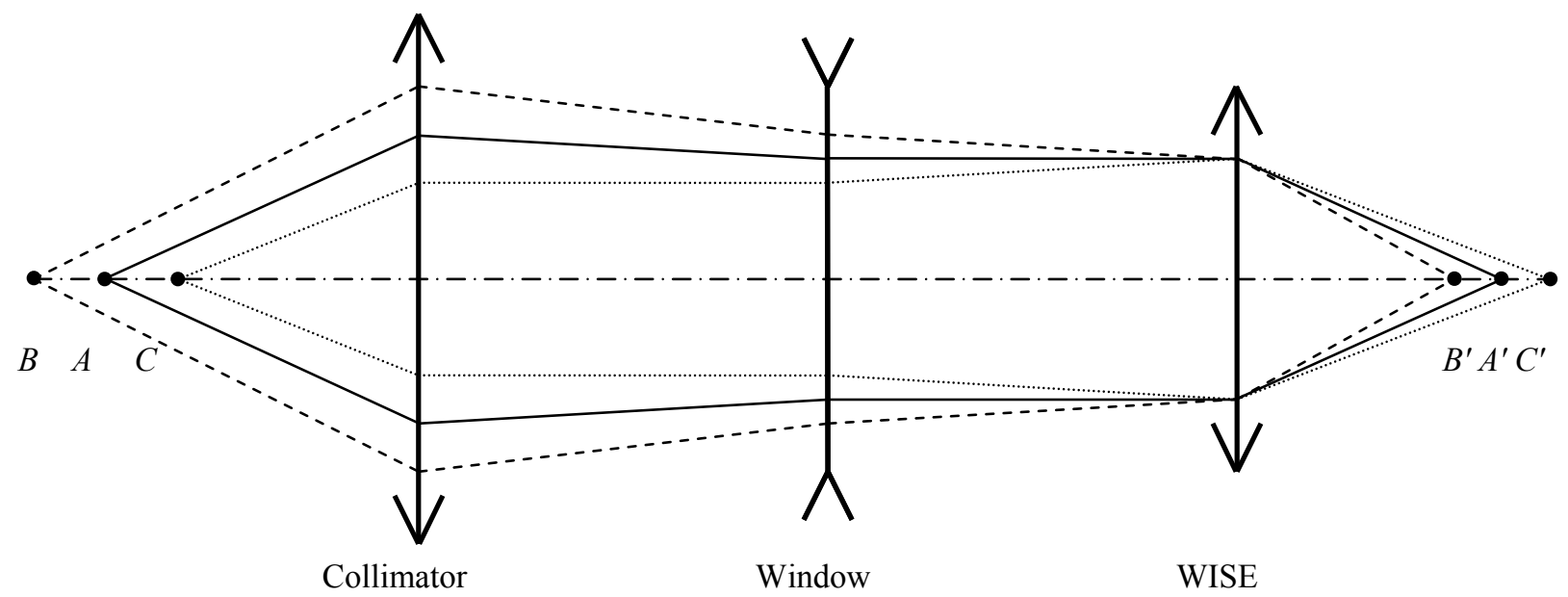

Figure 9. Conceptual ray trace of experimental setups used to make WISE focal plane measurements. SDL MIC2 focus measurements were made without a window.

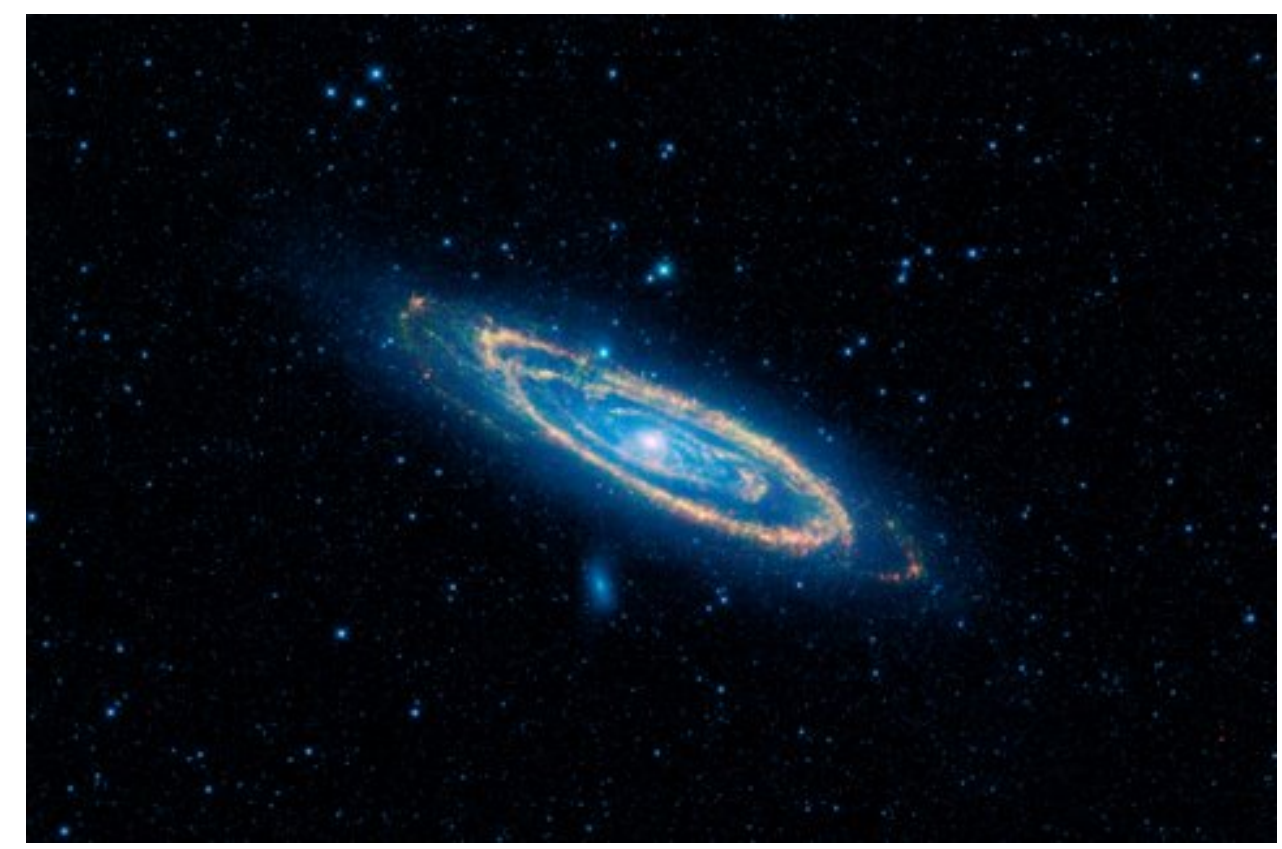

Figure 10. WISE pseudo-color image of Andromeda that illustrates that WISE is well focused. This image is a combination of images from all 4 WISE FPA's.

\section{CONCLUSIONS}

The WISE instrument met its image quality requirements with margin, in all 4 bands, indicating that the BSA worked as designed and that good focus was obtained in all 4 bands. This paper discusses the design and fabrication of the WISE beamsplitter assembly, which splits the optical output of the WISE telescope into 4 infrared bands and provides the means to set the focus of the 4 WISE instrument focal plane assemblies. Its large, heavy, rectangular, dichroic beamsplitters were successfully mounted to survive launch without significant distortion of their optical surfaces. This 
paper describes how fields of view of very complex instruments can be mapped into their focal plane assemblies by means of importing ray traces into mechanical solid models. This information is necessary to design wedge focal shims, such as those used to focus the WISE instrument. The WISE instrument was successfully focused on the ground from focus measurements made through a window with thermal gradients that produced a significant amount of defocus.

\section{REFERENCES}

1. Irace, W., Cutri, R., Duval,V., Eisenhardt, P., Elwell, J., Greanias, G., Heinrichsen, I., Howard, J., Lie, F., Royer, D. and Wright, E.L., "Managing the development of the Wide-field Infrared Survey Explorer mission," to be presented at SPIE Optics+Photonics Conference (2010).

2. Liu, F., Cutri, R., Greanias, G., Duval, V., Eisenhardt, P., Elwell, J. Heinrichsen, I., Howard, J., Irace, W., Mainzer, A., Razzaghi, A., Royer, D. and Wright E. L., "Development of the Wise-field Infrared Survey Explorer (WISE) mission", Proc. SPIE 7017 70170M (2008), doi:10.1117/12.790087.

3. Larsen, M. F., Latvakoski, H., Mainzer, A., Schick, S., and Drake, J., "Wide-field Infrared Survey Explorer science payload update," Proc. SPIE 7010 70100G (2008), doi:10.1117/12.789672. 